\title{
Estrutura populacional, aspectos da reprodução e alimentação dos Cyprinodontiformes (Osteichthyes) de um riacho do sudeste do Brasil ${ }^{1}$
}

\author{
José Marcelo Rocha Aranha ${ }^{2}$ \\ Érica Pellegrini Caramaschi ${ }^{3}$
}

\begin{abstract}
Population structure, reproductive aspects and feeding of Cyprinodontiformes fishes (Osteichthyes) from a coastal stream in southeastern Brazil. The population structure, reproductive aspects and feeding of Phalloceros caudimaculatus (Hensel, 1868), Poecilia vivipara Bloch \& Schneider (1801), Phalloptychus januarius (Hensel, 1868) e Jenynsia lineata (Jenyns, 1842) were studied in five sampling stations in the Ubatiba stream, municipality of Maricá (State of Rio de Janeiro, Brazil). In relation to the population structure, $P$. caudimaculatus was more representative upstream; $P$. vivipara mid-stream and downstream; $P$. januarius and $J$. lineata downstream. The species presented preponderance of females, except in the intermediate length classes. The occurence of imatures and females with young pointed out a long reproductive period and favoured areas for reproduction of the four species. The young forms of $P$. caudimaculatus were more abundant in station 1 (upstream), $P$. vivipara in station 4 (downstream) and $P$. januarius and $J$. lineata in station 5 (Maricá coastal lagoon). The higher occurences of females with young belong to $P$. caudimaculatus, in station 1 (upstream) and $P$. vivipara and $P$. januarius in station 5 (Maricá lagoon). The four studied species presented herbivorous diet and the differences found in the feeding may reflect the differences in the food availability in the microhabitats used by the species. So, we concluded that the studied species presented evident segregation in several levels of the different available resources in the environment.
\end{abstract}

KEY WORDS. Cyprinodontiformes, population structure, feeding, reproduction, coastal stream.

A influência das interações específicas na estrutura das comunidades e nas características morfológicas e comportamentais dos animais, ao longo do tempo evolutivo, tem suscitado debate pelo menos desde os tempos de Darwin. Alguns autores acreditam que a maioria das espécies vivem próximas da capacidade suporte do meio, tornando os recursos limitantes e, portanto, a competição seria um fator ecológico e evolutivo importante (e.g. HUNTCHINSON 1958; MACARTHUR 1972; SCHÖENER 1974; PIANKA 1982). Por outro lado, outros autores acreditam que a ação desestabilizadora de fatores físicos manteria as populações abaixo da capacidade de suporte, impedindo que os recursos se tornassem limitantes (e.g. ANDREWARTHA \& BIRCH 1954; WIENS 1977; CONNOR \& SIMBERLOFF 1979; STRONG 1980).

1) Parte do Convênio FINEP/UFRJ, processo 4.2.87.0588.00.

2) Departamento de Zoologia, Universidade Federal do Paraná. Caixa Postal 19020, 81531-990 Curitiba, Paraná, Brasil.

E-mail: jmaranha@bio.ufpr.br

3) Departamento de Ecologia, Instituto de Biologia, Universidade Federal do Rio de Janeiro. Caixa Postal 68020, 20941-970, Rio de Janeiro, Rio de Janeiro, Brasil. 
HUNTCHINSON (1959) afirma que a seleção natural, juntamente com o isolamento e subseqüente invasão mútua de áreas, tem levado à evolução das espécies simpátricas que, no equilíbrio, ocupam nichos diferentes. Deste modo, as diferenças de nicho minimizariam, através da partilha de recursos, a competição (DIAMOND 1978).

SCHÖENER (1974) afirma que a partilha de recursos pode ocorrer em diferentes dimensões deste nicho. Para ele, a alta sobreposição em uma dimensão implicaria em baixa similaridade em outras, preservando um nível mínimo de segregação na utilização dos recursos. O mesmo autor afirma que as três dimensões do nicho mais importantes para as espécies são a espacial, a temporal e a trófica.

Várias críticas são feitas à teoria de partilha de recursos. WIENS (1977) e GROSSMAN et al. (1982) propõem que a alta variabilidade ambiental impede o estado de equilíbrio nas comunidades, tornando a competição um fenômeno menos intenso e de difícil mensuração. Por outro lado, autores favoráveis à teoria de partilha de recursos têm refutado as críticas insistentemente, pois concordam com a importância da influência da variação ambiental no tamanho das populações, mas discordam que isto reduziria o papel das interações biológicas (e.g. YANT et al. 1984).

O número de trabalhos com peixes abordando a partilha de recursos nas três dimensões (espacial, temporal e trófica) aumentou muito nos últimos anos (Ross 1986), embora poucos trabalhos tenham sido feitos com Cyprinodontiformes (KEAST 1978; WERNER et al. 1978)

Estudos envolvendo recursos e taxocenoses de peixes em riachos do Brasil são escassos (e.g. ARANHA et al. 1993). Em riachos litorâneos, CosTA (1987), SABINO \& CASTRO (1990) e ARANHA \& CARAMASCHI (1997) abordam a alimentação e/ou ocupação espacial de diferentes espécies.

O objetivo deste trabalho é analisar a estrutura das populações e as relações alimentares e reprodutivas envolvidas na coexistência das quatro espécies de Cyprinodontiformes que ocorrem em um riacho litorâneo do sudeste brasileiro: Phalloceros caudimaculatus (Hensel, 1868), Poecilia vivipara Bloch \& Schneider (1801), Phalloptychus januarius (Hensel, 1868) e Jenynsia lineata (Jenyns, 1842).

\section{MATERIAL E MÉTODOS}

Foi adotada como área de estudo a drenagem do rio Ubatiba (Maricá, Rio de Janeiro, Brasil) (22 $\left.55^{\prime} \mathrm{S}, 42^{\circ} 49^{\prime} \mathrm{W}\right)$. Este rio tem aproxidamente $15 \mathrm{~km}$ de extensão e desemboca no sistema lagunar de Maricá. O percurso envolve um trecho de serra, onde ocorre vegetação marginal arbórea e leito de pedras e areia e um trecho de baixada com vegetação marginal arbustiva e fundo areno-lodoso.

Foram definidas quatro estações de amostragens numeradas em ordem crescente ao longo do rio Ubatiba e a quinta na lagoa de Maricá. A estação 1 era localizada no trecho superior da bacia, a estação 2 no trecho médio, a estação 3 no trecho médio-inferior, a 4 no trecho inferior e a 5, na lagoa próximo à desembocadura do rio. Em ARANHA \& CARAMASCHI (1997) há um mapa esquemático e o grau de similaridade das estações e da metodologia de coleta. 
Os peixes foram coletados mensalmente, nas estações 1 a 4, de junho de 1987 a julho de 1988, utilizando-se peneira e rede de malha fina. A estação 5 foi amostrada de julho de 1987 a julho de 1988. Exemplares testemunho foram depositados no Museu Nacional, Rio de Janeiro, Brasil, sob os números MNRJ12252 a 12255.

Os peixes foram fixados em mistura de Transeau (três partes de álcool $96^{\circ} \mathrm{GL}$, uma parte de formol e seis partes de água) que fixa bem os peixes e, ao mesmo tempo, preserva as algas do conteúdo estomacal. Depois de alguns dias, os peixes foram transferidos para álcool a $70^{\circ} \mathrm{GL}$, onde foram conservados. Foram então medidos quanto ao comprimento total e agrupados em classes de comprimento de 0,5 centímetro e examinados quanto ao sexo (pela presença de gonopódio).

Os exemplares da classe 1,6 a 2,0 cm ou menores e que não apresentavam indício de desenvolvimento de gonopódio ou de atividade reprodutiva foram classificados como jovens.

As fêmeas foram examinadas macroscopicamente quanto ao estado de maturação sexual, pois as fêmeas chamadas grávidas apresentam a região ventral abaulada. Nos casos de dúvida, foi feita uma pequena incisão e foram chamadas de grávidas as fêmeas que apresentavam embriões em desenvolvimento. Por outro lado, as que não apresentavam a região ventral abaulada ou nas quais não foram observados embriões, foram chamadas de não-grávidas.

Para a caracterização da estrutura populacional foram utilizados dados de 702 Phalloceros caudimaculatus, 1608 de Poecilia vivipara, 1221 de Phalloptychus januarius e 121 de Jenynsia lineata.

A época reprodutiva foi analisada pela freqüência relativa mensal de fêmeas grávidas e de jovens na população para cada espécie em cada estação de coleta, ao longo do período estudado. Estes resultados foram testados estatisticamente, quando possível, pela prova do Qui-quadrado $\left(X^{2}\right)$ para k amostras independentes (SIEGEL 1975), para determinar a significância estatística da diferença nas ocorrências de fêmeas grávidas e não grávidas e de jovens e adultos em relação aos bimestres. Assim, $o X^{2}$ significativo indicaria relação entre a ocorrência de fêmeas grávidas ou de jovens e os bimestres e confirma o que chamamos de "tendência à sazonalidade".

Para o estudo do hábito alimentar, foram analisados exemplares das espécies consideradas "constantes" em cada estação (ARANHA \& CARAMASCHI 1997), exceto a estação 4 que apresentava muita semelhança com a estação 5. Foi examinado o conteúdo do trato digestivo de 10 indivíduos de $P$. caudimaculatus da estação 1 e 6 da estação 3. De $P$. vivipara foram analisados 10 exemplares da estação 2,10 da 3 e 10 da 5 . De $P$. januarius e de $J$. lineata foram examinados, respectivamente, 10 exemplares de cada da estação 5 .

Para o estudo da variação interespecífica na composição da dieta em relação ao tamanho, foram examinados 10 exemplares adicionais de $P$. caudimaculatus da estação 1 , de $P$. vivipara da estação 3 , de $P$. januarius e $J$. lineata da 5 , totalizando 20 exemplares para cada espécie nessas estações. No total, 10 exemplares pertenciam à classe de comprimento $1,6-2,0 \mathrm{~cm}$ (a partir daqui nomeada classe 1) e 10 à classe 3,6-4,0 cm (classe 2). Estas classes de comprimento foram escolhidas por constituírem a menor e a maior dentre as classes de comprimento de maior freqüência. 
Cada exemplar foi dissecado e da porção anterior do trato digestivo foram extraídas secções de $1,0 \mathrm{~cm}$, sendo que dos exemplares da classe 1 foi retirada uma secção e dos exemplares da classe 2, duas secções. Cada secção foi lavada em álcool a $70^{\circ} \mathrm{GL}$ sobre lâmina de microscopia, coberta com lamínula e examinada em microscópio óptico. Os itens do conteúdo estomacal foram contados para estimar a importância numérica de cada item na dieta pelo método numérico adaptado para o presente estudo como descrito em ARANHA (1993). Feito isto, foram examinados todos os campos da lamínula e os itens que eventualmente não tivessem sido observados foram registrados para análise qualitativa pelo método de freqüência de ocorrência (HYSLOP 1980).

O material do conteúdo do trato digestivo foi determinado no menor nível taxonômico possível com base em Bourrelly $(1968,1970,1972)$ e Bicudo \& BICUDO (1970) para as algas e, para os itens de origem animal, NEEDHAM \& NEEDHAM (1978) e MACAN (1959).

Os resultados foram analisados utilizando-se o Índice Alimentar (KAWAKAMI \& VAZZOLER 1980).

Para a análise da sobreposição alimentar foi utilizada a fórmula de Morisita modificada por HORN (1972), conforme apresentada por SMITH \& ZARET (1982), utilizando os valores do Índice Alimentar de cada item do conteúdo estomacal. A matriz de similaridade foi aplicada a análise de agrupamento UPGMA (ROMESBURG 1990).

\section{RESULTADOS}

\section{Estrutura da População}

$\mathrm{O}$ comprimento total de $P$. caudimaculatus variou da classe $0,6-1,0 \mathrm{~cm}$ à $4,6-5,0 \mathrm{~cm}$, sendo que $61,5 \%$ dos exemplares pertenciam às classes $1,6-2,0 \mathrm{~cm}$ a 2,6-3,0 cm. A única estação onde foram coletados exemplares de todas as classes de comprimento foi a 1 .

$P$. vivipara variou da classe $0,6-1,0 \mathrm{~cm}$ a $7,1-7,5 \mathrm{~cm}$ sendo que as classes melhor representadas foram $1,1-1,5 \mathrm{~cm}$ a $3,0-3,5 \mathrm{~cm}$ com $74,9 \%$ dos exemplares da espécie coletados. Na estação 4 houve grande predomínio da classe 1,1-1,5cm (46\% dos exemplares coletados na estação) e na estação 5 predominaram as classes $3,6-4,0 \mathrm{~cm}$ a $5,1-5,5 \mathrm{~cm}$ ( $59 \%$ dos exemplares coletados).

Os exemplares de $P$. januarius variaram de $1,1-1,5 \mathrm{~cm}$ a $4,6-5,0 \mathrm{~cm}$ sendo que $65,4 \%$ dos indivíduos pertenciam às classes $1,6-2,0 \mathrm{~cm}$ a $2,6-3,0 \mathrm{~cm}$. $J$. lineata variou de $1,1-1,5 \mathrm{~cm}$ a $6,6-7,0 \mathrm{~cm}$ com predomínio das classes $1,6-2,0 \mathrm{~cm}$ a 3,1-3,5 $\mathrm{cm}$ com $74,4 \%$. A estação onde ambas as espécies foram melhor representadas foi a 5 , ou seja, na lagoa.

Todas as espécies estudadas apresentaram uma amplitude de variação das classes de comprimento muito menor em machos que em fềmeas e um predomínio de fêmeas sobre machos (Fig. 1).

A análise da proporção sexual por classe de comprimento mostra que há predomínio de fêmeas nas classes menores e maiores (Fig. 1). Nas classes de comprimento intermediárias, ou a proporção macho:fêmea não difere significativamente de 1:1 ou há predomínio estatisticamente significativo de fêmeas (Fig. 1). 

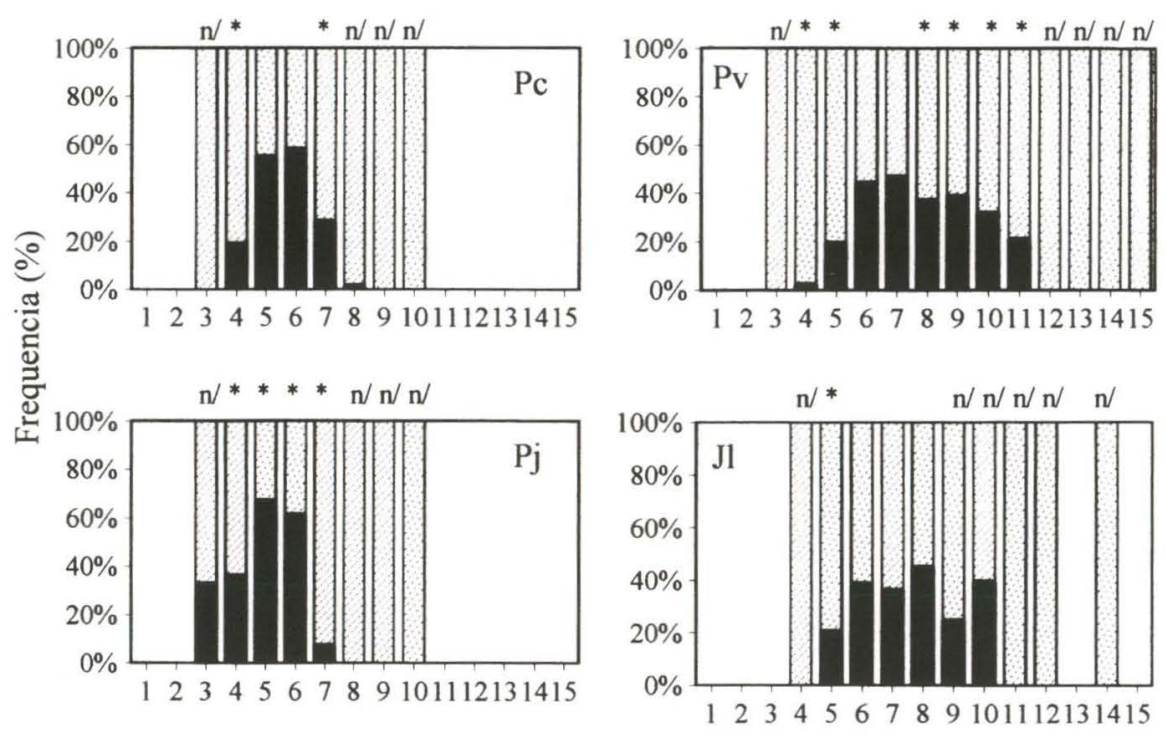

Classes de Comprimento

Machos $\square$ Femeas

Fig. 1. Freqüência relativa de ocorrência de machos e fêmeas de Phalloceros caudimaculatus (Pc), Poecilia vivipara (Pv), Phalloptychus januarius (Pj) e Jenynsia lineata (JI) em cada classe de comprimento no periodo estudado. $\left({ }^{*}\right) X^{2}$ significativo com $\alpha=0,05 ; \mathrm{n} /$ : cálculo do $X^{2}$ impossível.

Quanto à proporção entre jovens e adultos na população, houve predomínio de adultos nas quatro espécies (Tab. I). P. caudimaculatus apresentou evidente predomínio de adultos, principalmente na estação 3 (Tab. I). A maior ocorrência de jovens de $P$. caudimaculatus foi na estação 1 com $15,6 \%$ (Tab. I).

A ocorrência de jovens variou sazonalmente sendo que nos bimestres outubro-novembro e dezembro-janeiro houve aumento de jovens na amostra total e na estação 1 (Fig. 2).

A ocorrência de jovens de $P$. vivipara foi de $22,4 \%$ do total de exemplares coletados (Tab. I). Na estação 4 houve grande predomínio de jovens $(52,7 \%)$ enquanto nas estações 2, 3 e 5 o predomínio foi de adultos (Tab. I).

Ao longo do ano, a ocorrência de jovens desta espécie foi mais alta nos bimestres agosto-setembro, outubro-novembro e dezembro-janeiro (Fig. 2).

Em $P$. januarius a ocorrência de jovens foi muito pequena $(6,3 \%$ dos exemplares) e praticamente só na estação 5 (Tab. I). Ao longo do ano, jovens ocorreram nos bimestres outubro-novembro, dezembro-janeiro, abril-maio e junhojulho/88 (Fig. 2).

Quanto à $J$. lineata, $14,9 \%$ da amostra total foi de jovens e $94,4 \%$ deles ocorreu na estação 5 (Tab. I). Para esta espécie, jovens ocorreram nos bimestres agosto-setembro, outubro-novembro, abril-maio e junho-julho/88 (Fig. 2). 
Tabela I. Distribuição das freqüência relativas de jovens, adultos, fêmeas grávidas e não grávidas de $P$. caudimaculatus, $P$. vivipara, $P$. januarius e J. lineata para cada estação de coleta e para a amostra total.

\begin{tabular}{|c|c|c|c|c|c|c|c|}
\hline \multirow{2}{*}{ Espécies } & & \multicolumn{6}{|c|}{ Estaçăo de coleta } \\
\hline & & 1 & 2 & 3 & 4 & 5 & Total \\
\hline \multirow{4}{*}{$\begin{array}{l}\text { Phalloceros } \\
\text { caudimaculatus }\end{array}$} & Jovens & 15,6 & 10,0 & 2,9 & - & - & 13,0 \\
\hline & Adultos & 84,4 & 90,0 & 97,1 & - & - & 87,0 \\
\hline & Fêmea grávida & 45,1 & 14,3 & 12,5 & - & - & 37,8 \\
\hline & Fêmea não grávida & 54,9 & 85,7 & 87,5 & - & - & 62,2 \\
\hline \multirow{4}{*}{$\begin{array}{l}\text { Poecilia } \\
\text { vivipara }\end{array}$} & Jovens & - & 11,0 & 13,6 & 52,7 & 4,5 & 22,4 \\
\hline & Adultos & - & 89,0 & 86,4 & 47,3 & 95,5 & 77,6 \\
\hline & Fêmea grávida & - & 27,1 & 21,2 & 19,6 & 49,6 & 26,8 \\
\hline & Fêmea não grávida & - & 72,9 & 78,8 & 80,4 & 50,4 & 73,2 \\
\hline \multirow{4}{*}{$\begin{array}{l}\text { Phalloptychus } \\
\text { januarius }\end{array}$} & Jovens & - & - & - & 1,5 & 6,6 & 6,3 \\
\hline & Adultos & - & - & 100,0 & 98,5 & 93,4 & 93,7 \\
\hline & Fêmea grávida & - & - & - & 5,3 & 48,3 & 45,8 \\
\hline & Fèmea năo grávida & - & - & 100,0 & 94,7 & 51,7 & 54,2 \\
\hline \multirow{4}{*}{$\begin{array}{l}\text { Jenynsia } \\
\text { lineata }\end{array}$} & Jovens & - & - & - & 10,0 & 15,6 & 14,9 \\
\hline & Adultos & - & - & 100,0 & 90,0 & 84,4 & 85,1 \\
\hline & Fêmea grávida & - & - & - & 50,0 & 14,5 & 16,2 \\
\hline & Fêmea năo grávida & - & - & 100,0 & 50,0 & 85,5 & 83,8 \\
\hline
\end{tabular}
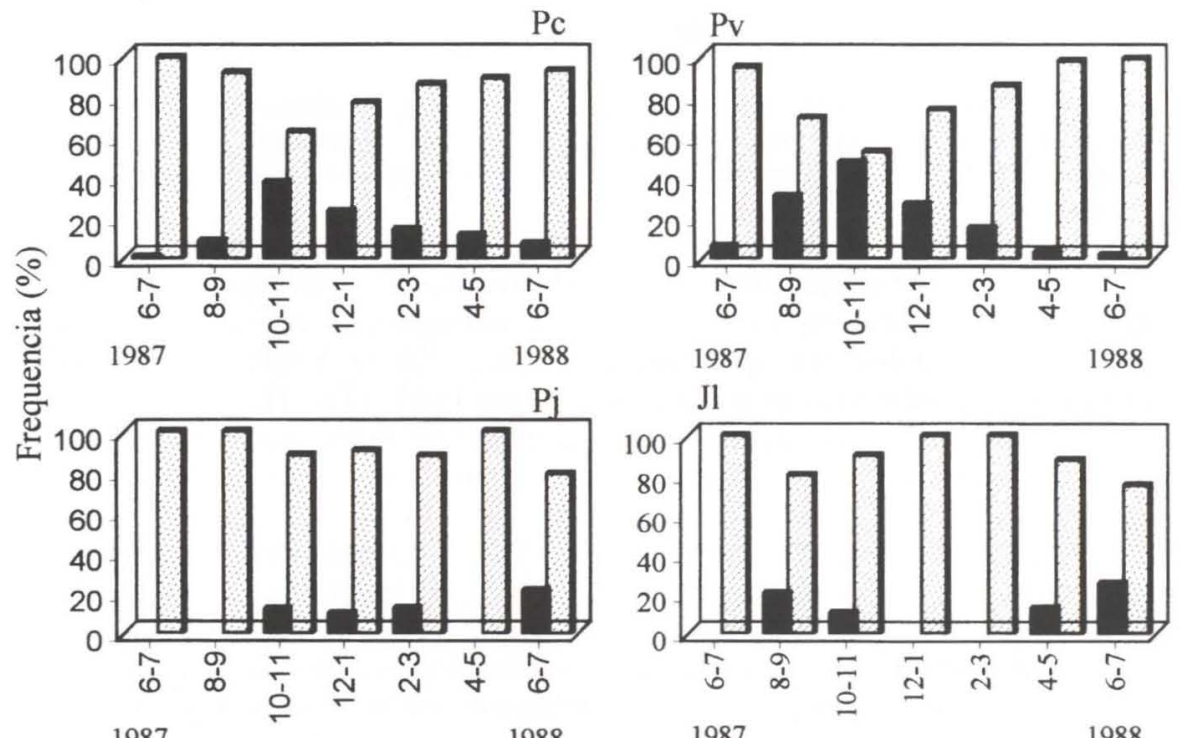

1987

1988

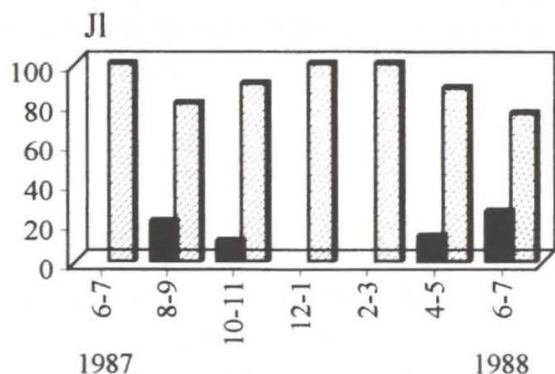

Bimestres

Jovens $\square$ Adultos

Fig. 2. Freqüência relativa de ocorrência de jovens e adultos de Phalloceros caudimaculatus $(\mathrm{Pc})$, Poecilia vivipara (Pv), Phalloptychus januarius (Pj) e Jenynsia lineata (JI) por bimestre no período estudado. 


\section{Reprodução}

Nas espécies estudadas houve predomínio de fêmeas não grávidas sobre fêmeas grávidas (Tab. I). Quanto à ocorrência de fêmeas grávidas ao longo do período estudado, houve tendência à sazonalidade nas quatro espécies (Phalloceros caudimaculatus: $X^{2}=41,86, \mathrm{P}<0,05$; Poecilia vivipara: $X^{2}=118,84, \mathrm{P}<0,05$; Phalloptychus januarius: $X^{2}=145,34, P<0,05$; Jenynsia lineata: $X^{2}=20,43$, $\mathrm{P}<0,05$ ).

As fêmeas grávidas de $P$. caudimaculatus predominaram na estação 1 ( $92,4 \%$ do total de fêmeas grávidas coletadas) e nos bimestres agosto-setembro, outubro-novembro, dezembro-janeiro e abril-maio (Fig. 3). Nas estações 2 e 3 houve grande predomínio de fêmeas não grávidas.

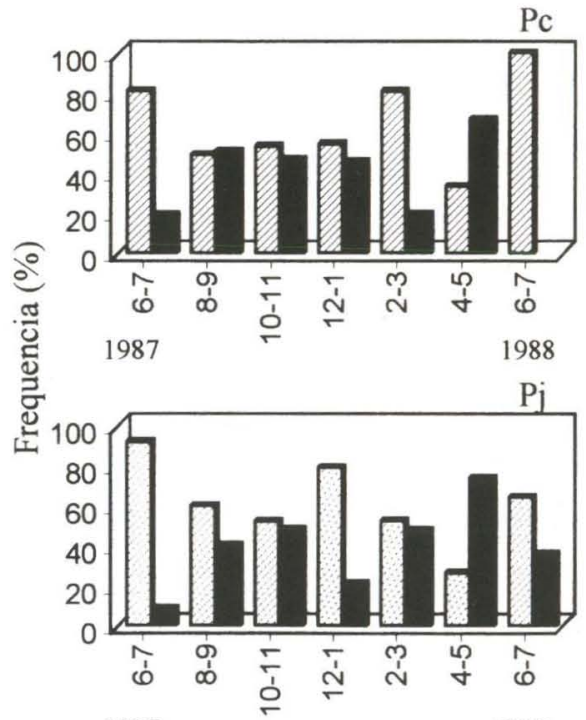

1987

1988
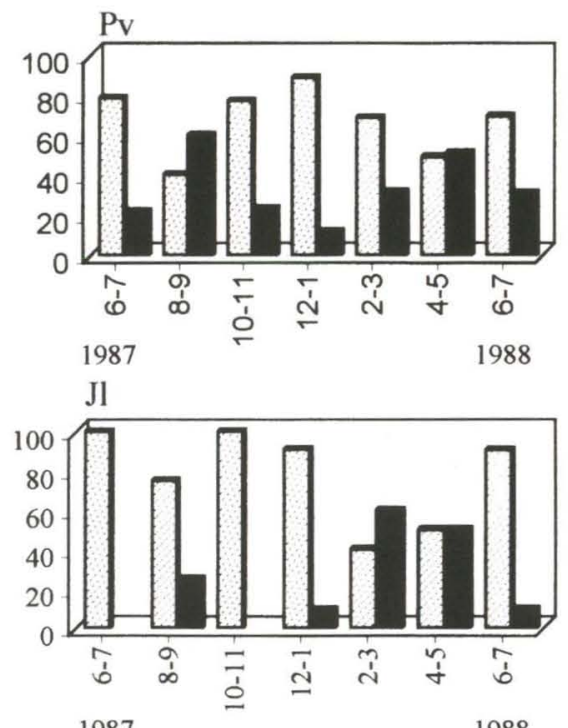

Bimestres

口Femeas n/ Gravidas $D$ Femeas Gravidas

Fig. 3. Freqüência relativa de ocorrência de fêmeas não grávidas e grávidas de Phalloceros caudimaculatus (Pc), Poecilia vivipara (Pv), Phalloptychus januarius (Pj) e Jenynsia lineata (JI) por bimestre no período estudado.

Para P. vivipara, a maior ocorrência de fêmeas grávidas ocorreu na estação 5 (Tab. I) e nos bimestres agosto-setembro, abril-maio e Junho-Julho/88 (Fig. 3). Nas estações 2, 3, e 4 houve predomínio de fêmeas não grávidas se comparadas com a estação 5 (Tab. I).

P. januarius foi a espécie onde o predomínio de fêmeas não grávidas foi menor (Tab. I). A ocorrência de fêmeas grávidas foi de $45,8 \%$ do total de fêmeas, sendo que $99,4 \%$ destas fêmeas grávidas ocorreram na estação 5 (Tab. I). Ao longo 
de todo o ano foram coletadas fêmeas grávidas mas os bimestres com maior ocorrência foram agosto-setembro, outubro-novembro, fevereiro-março e junho-julho/88 (Fig. 3).

A freqüência de coleta de fềmeas grávidas de $J$. lineata foi muito pequena $(16,2 \%$ do total; Tab. I). Assim, apesar de ter sido encontrada tendência à sazonalidade $\left(X^{2}=20,43, \mathrm{P}<0,05\right)$, o pequeno número de fêmeas grávidas torna difícil a definição da estação reprodutiva (Fig. 3).

\section{Alimentação}

Os peixes estudados apresentaram grande diversidade na dieta, totalizando 32 categorias de itens alimentares (Tab. II). Os itens vegetais foram determinados até o nível de família considerando a forma da célula.

Dois itens, helminto não identificado e tardígrado, ocorreram apenas na análise qualitativa em $P$. vivipara da classe 2 na estação $3(10 \%$ e $20 \%$, respectivamente) e foram retirados da tabela II.

Tabela II. Valores do Índice Alimentar de cada item do conteúdo do trato digestivo de $P$. caudimaculatus, $P$. vivipara, $P$. januarius e $J$. lineata das classes de comprimento estudadas (cl 1 e 2) nas estações 1, 2, 3 e 5 (est 1, 2, 3 e 5, respectivamente) do rio Ubatiba. (n/l) Não identificado, (*) valor menor que 0,01 .

\begin{tabular}{|c|c|c|c|c|c|c|c|c|c|c|c|}
\hline \multirow{2}{*}{ Itens } & \multicolumn{3}{|c|}{ P. caudimaculatus } & \multicolumn{4}{|c|}{$P$. vivipara } & \multicolumn{2}{|c|}{$P$. januarius } & \multicolumn{2}{|c|}{ J. lineata } \\
\hline & $\begin{array}{r}\mathrm{Cl} 1 \\
\text { Est } 1\end{array}$ & $\begin{array}{r}\mathrm{Cl} 2 \\
\text { Est } 1\end{array}$ & $\begin{array}{r}\mathrm{Cl} 1 \\
\text { Est } 3\end{array}$ & $\begin{array}{r}\mathrm{Cl} 1 \\
\text { Est } 2\end{array}$ & $\begin{array}{r}\mathrm{Cl} 1 \\
\text { Est } 3\end{array}$ & $\begin{array}{r}\mathrm{Cl} 2 \\
\text { Est } 3\end{array}$ & $\begin{array}{r}\mathrm{Cl} 2 \\
\text { Est } 5\end{array}$ & $\begin{array}{r}\mathrm{Cl} 1 \\
\text { Est } 5\end{array}$ & $\begin{array}{r}\mathrm{Cl} 2 \\
\text { Est } 5\end{array}$ & $\begin{array}{r}\mathrm{Cl} 1 \\
\text { Est } 5\end{array}$ & $\begin{array}{r}\mathrm{Cl} 2 \\
\text { Est } 5\end{array}$ \\
\hline Achnantaceae & 0,18 & 0,23 & 0,00 & 0,00 & 0,50 & 0,37 & 0,00 & 0,27 & 0,00 & 0,00 & 0,00 \\
\hline Anaulaceae & 0,00 & 0,00 & 0,00 & 0,00 & 0,00 & 0,00 & 38,09 & 1,68 & 7.37 & 7.92 & 14,76 \\
\hline Coscinodiscaceae & 0,68 & 3,21 & 0,00 & 0,00 & 1,53 & 1,76 & 1,42 & 0,00 & 0,00 & 1,97 & 0,00 \\
\hline Diatomaceae & 0,61 & 1,84 & 0,00 & 2,85 & 0,10 & 0,05 & 0,00 & 0,00 & 0,00 & 0,98 & 0,00 \\
\hline Naviculaceae & 17,04 & 29,26 & 94,02 & 47,62 & 68,12 & 67,97 & 6,43 & 0,55 & 1,29 & 14,84 & 6,70 \\
\hline Nitzschiaceae & 0,27 & 0,08 & 0,00 & 0,71 & 0,32 & 0,16 & 0,00 & 0,00 & 0,00 & 0,00 & 0,00 \\
\hline Surirelaceae & 0,04 & 0,25 & 0,05 & 0,00 & 0,50 & 0,22 & 0,00 & 0,27 & 0,00 & 0,49 & 2,66 \\
\hline Bacillariophyceae (n/l) & 8,83 & 13,09 & 0,00 & 1,90 & 14,54 & 12,89 & 1,07 & 0,27 & 0,00 & 0,00 & 0,00 \\
\hline Oocystaceae & 1,40 & 2,65 & 0,52 & 0,00 & 4,11 & 6,17 & 0,00 & 0,00 & 0,00 & 0,00 & 0,00 \\
\hline Scenedesmaceae & 0,00 & 0,00 & 0,00 & 0,00 & 0,00 & 0,00 & 17,17 & 95,24 & 56,99 & 0,00 & 0,00 \\
\hline Chlorophyceae $(n / l)$ & 13,94 & 24,37 & 0,00 & 0,00 & 0,00 & 0,20 & 0,00 & 0,00 & 0,00 & 39,61 & 3,35 \\
\hline Oscillatoriaceae & 53,34 & 17,18 & 2,13 & 0,00 & 6,95 & 9,44 & 0,00 & 0,00 & 0,00 & 0,00 & 0,00 \\
\hline Euglenaceae & 3,05 & 0,17 & 1,43 & 0,71 & 0,10 & 0,00 & 0,71 & 0,00 & 0,00 & 0,00 & 0,00 \\
\hline Desmidiaceae & 0,06 & 0,70 & 1,74 & 8,33 & 0,72 & 0,40 & 1,07 & 0,55 & 0,00 & 0,00 & 0,00 \\
\hline Mesoteniaceae & * & 6,06 & 0,00 & 0,00 & 0,00 & 0,00 & 0,00 & 0,00 & 0,00 & 0,00 & 0,00 \\
\hline Filamentosa $(\mathrm{n} / \mathrm{l})$ & 0,31 & 0,22 & 0,00 & 0,00 & 0,03 & 0,01 & 0,00 & 0,00 & 0,00 & 0,00 & 0,00 \\
\hline Algas $(n / l)$ & $*$ & 0,18 & 0,02 & 0,71 & 0,00 & 0,11 & 4,29 & 0,55 & 29,14 & 17,32 & 0,00 \\
\hline Material vegetal $(n / l)$ & 0,15 & 0,07 & 0,05 & 0,00 & 0,37 & 0,15 & 0,00 & 0,00 & 0,00 & 0,00 & 0,00 \\
\hline Protista & $*$ & $*$ & 0,00 & 0,00 & 0,00 & 0,00 & 0,00 & 0,00 & 0,00 & 0.00 & 0,00 \\
\hline Rotifero & 0,00 & 0,00 & 0,00 & 0,00 & 0,00 & * & 0,00 & 0,00 & 0,00 & 0,00 & 0,00 \\
\hline Nematóide & 0,00 & 0,00 & 0,00 & 0,00 & 0,00 & $*$ & 0,00 & 0,00 & 0,00 & 0,00 & 0,33 \\
\hline Microcrustáceo & 0,00 & 0,00 & 0,00 & 0,00 & 0,01 & 0,00 & 0,00 & 0,00 & 0,00 & 0,00 & 0,00 \\
\hline Crustáceo $(n / l)$ & 0,00 & 0,00 & 0,00 & 0,00 & 0,00 & 0,00 & 0,03 & 0,00 & 0,00 & 4,94 & 0,00 \\
\hline Larva Chironomidae & 0,00 & 0,00 & 0,00 & 35,01 & 0,00 & 0,00 & 0,00 & 0,00 & 0,00 & 0,00 & 0,00 \\
\hline Hymenoptera & 0,00 & 0,00 & 0,00 & 0,00 & 0,00 & 0,00 & 0,00 & 0,00 & 0,00 & 0,00 & 0,33 \\
\hline Ácaro & 0,00 & 0,00 & 0,00 & 0,23 & 0,00 & 0,00 & 0,00 & 0,00 & 0,00 & 0,00 & 0,33 \\
\hline Fragmento de artropodo & 0,01 & * & 0,00 & 1,90 & 0,00 & 0,00 & 0,00 & 0,00 & 0,00 & 5,93 & 8,05 \\
\hline Material Animal $(n / 1)$ & 0,00 & 0,00 & 0,00 & 0,00 & 0,00 & 0,01 & 0,00 & 0,00 & 0,00 & 0,00 & 0,00 \\
\hline Organismo $(\mathrm{n} / \mathrm{l})$ & 0,00 & 0,35 & 0,00 & 0,00 & 0,00 & 0,00 & 28,64 & 0,55 & 5,18 & 5,94 & 62,09 \\
\hline Material $(n / l)$ & 0,00 & 0,00 & 0,00 & 0,00 & 2,02 & 0,01 & 0,71 & 0,00 & 0,00 & 0,00 & 1,33 \\
\hline & 99,86 & 99,56 & 99,96 & 62,83 & 97,89 & 99,90 & 70,25 & 99,38 & 94,79 & 83,13 & 27,47 \\
\hline Material de origem animal & 0,01 & $*$ & 0,00 & 37,14 & 0,01 & 0,01 & 0,03 & 0,00 & 0,00 & 10.87 & 9,04 \\
\hline
\end{tabular}


As quatro espécies estudadas apresentaram dieta herbívora.

Na estação $1, P$. caudimaculatus foi estudado em duas classes de tamanho. Os principais itens encontrados no trato digestivo dos exemplares da classe 1 foram Oscillatoriaceae, Naviculaceae e Chlorophyceae não identificada (Tab. II). Para exemplares da classe 2, os principais itens foram Naviculaceae, Chlorophyceae, Oscillatoriaceae e Bacillariophyceae não idenficada (Tab. II). A análise de sobreposição alimentar indica $70 \%$ de sobreposição na dieta de $P$. caudimaculatus classes 1 e 2 pois, apesar de alguns dos principais itens terem sido os mesmos, as proporções foram diferentes (Fig. 4).

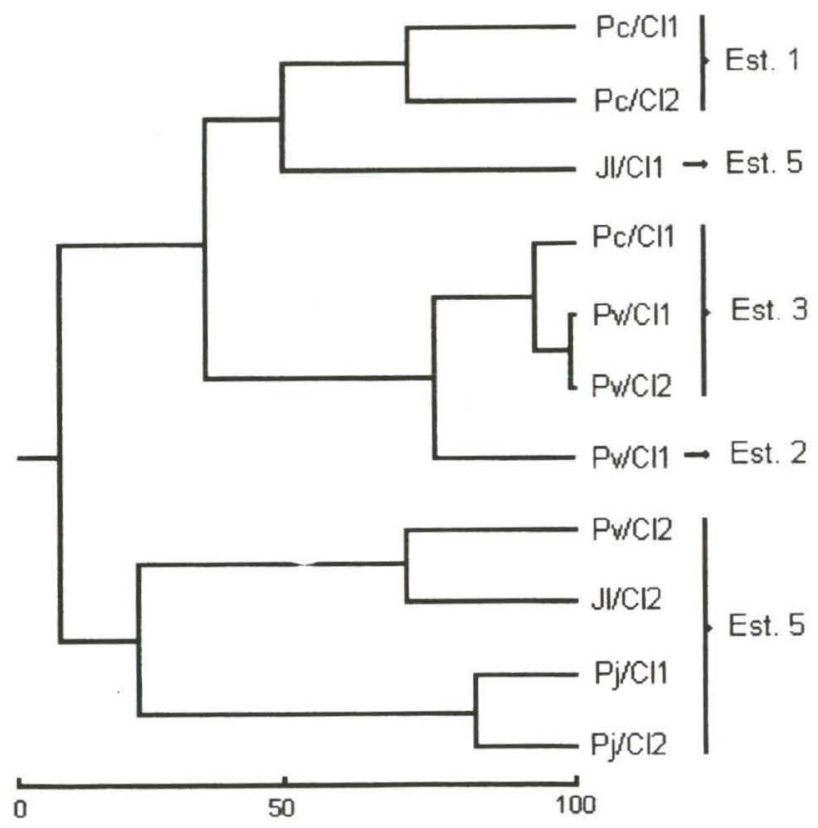

Fig. 4. Representação gráfica da análise de agrupamento das dietas de Phalloceros caudimaculatus ( $\mathrm{Pc}$ ), Poecilia vivipara (Pv), Phalloptychus januarius (Pj) e Jenynsia lineata (JI) nas estações 1, 2, 3 e 5 do rio Ubatiba, para individuos da classe de comprimento 1 (Cl1) e classe de comprimento $2(\mathrm{Cl} 2)$, no período estudado.

Na estação 2 foram estudados 10 exemplares da classe 1 de $P$._.vivipara e os principais itens foram Naviculaceae e larvas de Chironomidae (Tab. II).

Duas espécies foram estudadas na estação 3.P. caudimaculatus foi estudado apenas na classe 1 e o principal item foi Naviculaceae (Tab. II). É importante ressaltar que a diversidade de itens da dieta de $P$. caudimaculatus nesta estação é menor que na estação 1 e a de $P$. vivipara na estação 3. $P$. vivipara foi estudada em duas classes, sendo que os principais itens foram Naviculaceae e Baccilariophyceae não identificada para exemplares das classes 1 e 2 (Tab. II).

Pela análise de sobreposição alimentar, vê-se na figura 4 que $P$. vivipara da estação 2 apresentou maior semelhança com exemplares da mesma espécie das 
classes 1 e 2 e com exemplares de P. caudimaculatus, todos da estação 3. Entre os pecilídeos da estação 3 , a dieta de $P$. caudimaculatus foi $93 \%$ semelhante à dieta de $P$. vivipara tanto da classe 1 quanto da classe 2 (Fig. 4).

$\mathrm{Na}$ estação $5, P$. vivipara da classe 2 mostrou o predomínio dos itens Anaulaceae, Organismos não identificados e Scenedesmaceae na dieta (Tab. II). Scenedesmaceae foi o item mais importante para exemplares das duas classes de tamanho de $P$. januarius e o segundo item mais importante para exemplares da classe 2 foi "Algas não identificadas" (Tab. II). Para $J$. lineata, os itens mais importantes dos exemplares da classe 1 foram Chlorophyceae não identificadas, "Algas não identificadas" " "Naviculaceae". Para os exemplares da classe 2, os itens mais importantes foram "Organismos não identificados" e "Anaulaceae" (Tab. II).

Nesta estação, a análise de sobreposição mostra alta similaridade nas dietas de $P$. januarius das classe 1 e 2 com $82 \% ; P$. vivipara apresentou maior semelhança com a dieta de $J$. lineata classe $2(70 \%)$ e a dieta de $J$. lineata da classe 1 mostra similaridade muito baixa com a dieta dos outros guarus da mesma estação e $69 \%$ de semelhança com $P$. caudimaculatus da classe 2 da estação 1 (Fig. 4).

\section{DISCUSSÃO}

A estrutura em comprimento das espécies estudadas mostra que algumas populações estavam melhor representadas em determinadas estações de coleta, como o caso de $P$. can dimaculatus na estação 1 e de $P$. januarius e $J$. lineata na estação 5. Estes resultados reforçam as conclusões de ARANHA \& CARAMASCHI (1997) que apontam P. caudimaculatus como espécie de maior constância e freqüência no trecho superior do rio Ubatiba, $P$. vivipara nos trechos médio e inferior e $P$. januarius e $J$. lineata no trecho inferior.

Poecila vivipara mostrou predomínio de indivíduos pequenos na estação 4 e de exemplares maiores na estação 5. ARANHA \& CARAMASCHI (1997) afirmam que, nas estações 4 e 5 , P. vivipara, $P$. januarius $\mathrm{e} J$. lineata apresentaram ocupação espacial diferenciada em relação ao tamanho dos exemplares. Na estação 4 , exemplares maiores de $P$. vivipara (acima de $3 \mathrm{~cm}$ ) ocupavam o meio do rio em regiões mais profundas, junto ao substrato. Os indivíduos menores ocupavam áreas marginais, quase sem correnteza, junto à vegetação (ARANHA \& CARAMASCHI 1997). Como nesta estação não foi possível coletar todos os meses no microhabitat ocupado pelos indivíduos maiores, o predomínio dos exemplares menores provavelmente está superestimado por problemas de amostragem.

Quanto à proporção sexual, as espécies estudadas apresentaram predomínio de fêmeas, exceto quando considerados exemplares das classes de comprimento intermediárias. HENN (1916) aponta como geral o predomínio de fêmeas em coleções de pecilídeos. Alguns autores sugerem que a predação seletiva pode alterar a proporção sexual diminuindo a porcentagem de machos (KRUMHOLZ 1948) ou de fêmeas (BRITTON \& MOSER 1982). BOELY (1980, apud NARAHARA et al. 1985) afirma que a proporção sexual pode estar relacionada ao comprimento dos indivíduos. Provavelmente, as diferenças nas taxas de crescimento e mortalidade podem estar influenciando a proporção sexual dos pecilídeos estudados. 
No rio Ubatiba, as espécies estudadas parecem não empreender grandes deslocamentos (talvez apenas em curtos percursos), embora possam ocorrer deslocamentos involuntários rio abaixo durante trombas d'água. No entanto, a análise cuidadosa da ocorrência de jovens e fêmeas grávidas destas espécies permite a definição de áreas preferenciais de reprodução. As estações onde houve maior ocorrência de jovens foi a 1 para $P$. caudimaculatus, a 4 para $P$. vivipara e a 5 para $P$. januarius $\mathrm{e} J$. lineata. Quanto às fêmeas grávidas, as estações onde houve maior ocorrência foi a 1 para $P$._caudimaculatus e 5 para $P$. vivipara e $P$. januarius. Há uma clara concordância nestes resultados exceto para $P$. vivipara (maior ocorrência de jovens e fêmeas grávidas em diferentes estações) provavelmente pelos problemas de amostragem já discutidos. Além disso, há que se considerar que as estações $4 \mathrm{e}$ 5 são muito próximas (distante aproximadamente 500 metros) (ARANHA \& CARAMASCHI 1997). É possível que a ocorrência de jovens de $P$. caudimaculatus na estação 2 esteja superestimada ( $10 \%)$ pois o número de exemplares coletados nesta estação foi muito pequeno e, em termos absolutos, estes $10 \%$ correspondem a um único indivíduo. O mesmo problema pode ter ocorrido com J. lineata na estação 4 pois foram coletadas apenas quatro fêmeas, duas delas grávidas. Então, aparentemente os trechos com maior atividade reprodutiva foram o superior para $P$. caudimaculatus e o inferior para as outras espécies. Para $P$. vivipara houve também atividade reprodutiva no trecho médio mas, no trecho inferior esta foi aparentemente mais intensa.

A ovoviviparidade, característica comum das espécies estudadas, é reconhecida como uma estratégia que permite a reprodução com relativa independência das variações ambientais. A fertilização interna, segundo THIBAULT \& SCHULTZ (1978), pode permitir, entre outras coisas, a variação no intervalo dos períodos reprodutivos e tempo de gestação. A ocorrência de jovens e de fêmeas grávidas mostrou tendência à sazonalidade, pois os resultados foram significativos (exceto jovens de $J$. lineata). Entretanto, aparentemente a característica mais evidente foi que todas as espécies estudadas apresentaram um período reprodutivo bastante prolongado. KRAMER (1978) afirma que peixes de habitats tropicais estáveis tendem a ter períodos reprodutivos prolongados. Entretanto, MENEZES \& CARAMASCHI (1994) verificaram período reprodutivo prolongado para espécies de rios costeiros e atribuem esta estratégia a uma resposta biológica a ambientes instáveis, onde a reposição contínua de jovens constituiria um mecanismo de restabelecimento da estrutura da população. ARANHA \& CARAMASCHI (1997) mostraram que, após fortes chuvas, ocorre redução na abundância relativa e alterações na constância das espécies por estação. O periodo reprodutivo prolongado dessas espécies no rio Ubatiba sugere estratégia similar à descrita acima (MENEZES \& CARAMASCHI 1994).

Quanto à dieta, as quatro espécies estudadas apresentaram dieta herbívora, apesar de tradicionalmente os Cyprinodontiformes serem considerados insetívoros. SABINO \& CASTRO (1990) apresentam P. caudimaculatus como espécie onívora com tendência à herbivoria. Por outro lado, CosTA (1987) encontrou $100 \%$ de algas na dieta de $P$. caudimaculatus em rio costeiro próximo à lagoa de Saquarema (Rio de Janeiro) e TEIXEIRA (1989) 100\% de vegetal superior no conteúdo estomacal da mesma espécie em rio litorâneo do Rio Grande do Sul. 
Na estação $1, P$. caudimaculatus apresentou $70 \%$ de semelhança na dieta entre exemplares de menor porte (classe 1) e os de maior porte (classe 2). A maior ocorrência de Oscillatoriaceae em indivíduos menores pode ser reflexo de variação na distribuição do item no ambiente ou de seletividade, por parte dos peixes, na captura deste item. PAYNE (1986) cita Oscillatoria como planctônica e, desta forma, a correnteza poderia influenciar na abundância em diferentes habitats tornando a disponibilidade deste item diferenciada. Por outro lado, vários autores têm observado seletividade em função do tamanho dos exemplares na captura de itens em peixes planctófagos (e.g. EGGERS 1977).

Foram observados para $P$. vivipara, na estação 2 , os menores valores de índice alimentar de itens de origem vegetal $(62,83 \%$ no total) e os maiores valores de itens de origem animal $(37,14 \%$ no total), comparando-se com a dieta dos exemplares da mesma espécie nas estações 3 e 5 . Isto pode ser reflexo das características ambientais da estação 2 pois, com o predomínio de forte correnteza (ARANHA \& CARAMASCHI 1997), itens como algas devem ser arrastados pela água, tornando a disponibilidade de itens alimentares diferente em relação à oferta alimentar nas estações 3 e 5. Esta variação no alimento disponível explicaria as diferenças na dieta de $P$. vivipara na estação 2 se comparado ao hábito alimentar da mesma espécie nas estações 3 e 5. ANGERMEIER \& KARR (1984) afirmam que a dieta em peixes reflete a preferência, a disponibilidade e o acesso ao alimento.

Na estação 3, Naviculaceae apresentou grande predomínio em P._caudimaculatus e $P$. vivipara, sugerindo grande abundância desta família de alga nesta estação.

A dieta dos exemplares de $P$. vivipara das duas classes de tamanho tiveram $100 \%$ de semelhança na estação 3. Os itens helmintos e nematóides não foram encontrados digeridos ou semi-digeridos e foram esporádicos, sugerindo constituírem-se de endoparasitas. SOARES-PORTO (1994) verificou a presença de nematóides parasitas no conteúdo estomacal de peixes do mesmo rio.

Na estação 5, foram analisados exemplares de $P$. vivipara, $P$. januarius e $J$. lineata. $\mathrm{O}$ total de itens contados em cada uma destas amostras foi muito baixo em comparação com os resultados obtidos nas outras estações. Este valor total baixo fez com que itens que ocorreram apenas uma vez atingissem freqüências maiores de $2 \%$ ao passo que, nas estações 1 e 3 , o item unitário representou menos de $0,1 \%$.

Os itens Scenedesmaceae e Anaulaceae ocorreram somente nos peixes coletados na estação 5 , sugerindo serem algas de distribuição restrita a esta estação.

Organismos não identificados ocorreram em alta frequêencia no conteúdo do trato digestivo de $P$. vivipara, $P$. januarius $\mathrm{e} J$. lineata na estação 5 . O trato digestivo de alguns exemplares destas espécies estava repleto deste item que parece ser constituído de ovos de algum invertebrado. SOARES-PORTO (1994) aponta a ocorrência de ovos no conteúdo estomacal de Pimelodella coletada no rio Ubatiba. Observamos neste estudo que estes ovos ocorreram principalmente nos exemplares de maior classe de tamanho, que procuram alimento no fundo, sugerindo a ocorrência deste item no fundo.

A comparação das dietas de $P$. januarius $\mathrm{e} J$. lineata mostra nítidas diferenças. Phalloptychus januarius alimenta-se preferencialmente de Scenedesmaceae, item que foi ocasional em J. lineata. Por outro lado, J. lineata ingeriu Chlorophyta 
não identificada em abundância e este item sequer ocorreu em $P$. januarius. Similaridades nas dietas dos peixes estudados na estação 5 foram as menores encontradas. $P$. vivipara apresentou $7 \%$ de similaridade com $J$. lineata da classe 2 , provavelmente pela ocorrência importante de Anaulaceae e Organismos não identificados. Exemplares da classe 1 de $J$. lineata mostraram maior semelhança com $P$. caudimaculatus da classe 2 na estação 1 , provavelmente pela ocorrência de Chlorophyceae não identificada e Naviculaceae. Estes dados sugerem possível partilha alimentar intra e interespecífica nesta estação 5. Ross (1986) em trabalho de revisão sobre partilha de recursos, afirma que $64 \%$ das pesquisas de partilha com mais de duas espécies de peixes apontam a segregação a nível alimentar como a mais importante.

Deste modo, conclui-se que as espécies estudadas apresentaram evidências de segregação em diversos níveis dos recursos disponíveis no ambiente. $P$. caudimaculatus e $P$. vivipara apresentaram partilha a nivel espacial (ARANHA \& CARAMASCHI 1997), sendo que os resultados obtidos para $P$. caudimaculatus na estação 3 sugerem que esta população possivelmente seja prejudicada pela simpatria com $P$. vivipara. Os resultados nas estações 4 e 5 indicam partilha espacial em relação ao tamanho (ARANHA \& CARAMASCHI 1997) e partilha trófica. Estas hipóteses necessitam de teste experimental, conforme sugere PRICE (1984) para estudos desta natureza. Por outro lado, são apoiadas por SCHÖENER (1974) quando este propõe importância crescente dos níveis espacial, trófico e reprodutivo da partilha. Ross (1986) afirma que, em estudos de partilha com mais de duas espécies de peixes de água doce, os níveis alimentar e espacial são os mais importantes.

Acreditamos que, apesar da evidente importância dos fatores abióticos na distribuição de populações de peixes em rios, os padrões observados para os Cyprinodontiformes do rio Ubatiba podem também ser influenciados pela partilha de recursos (trófico e espacial) e a predação.

\section{REFERÊNCIAS BIBLIOGRÁFICAS}

ANDREWARTHA, H.G. \& L.C. BiRCH. 1954. The distribution and abundance of animals. Univ. of Chicago Press, Chicago, 782p.

ANGermeIeR, P.I. \& J.R. KARR. 1984. Fish communities along enviromental gradients of tropical streams. Env. Biol. Fish. 9 (2): 39-57.

ARANHA, J.M.R. 1993. Método para análise quantitativa de algas e outros itens microscópicos de alimentação de peixes. Acta Biol. Paranaense, Curitiba, 22 $(1,2,3,4): 71-76$.

ARANHA, J.M.R.; E.P. CARAMASCHI \& U. CARAMASCHI. 1993. Ocupação ambiental, regime alimentar e época reprodutiva de duas espécies de Corydoras (Siluroidei, Callichthyidae) coexistentes no rio Alambari (Botucatu, SP). Revta bras. Zool. 10 (3): 453-466.

ARANHA, J.M.R. \& E.P. CARAMASCHI. 1997. Distribuição longitudinal e ocupação espacial de quatro espécies de Cyprinodontiformes no rio Ubatiba, Maricá, RJ. Acta Biol. Paranaense, Curitiba, $26(1,2,3,4):$ 125-140.

BICUDO, C.E.M. \& R.M.T. BICUDO. 1970. Algas de águas continentais continen- 
tais brasileiras. Chave ilustrada para identificação de gêneros. São Paulo, Fundação Brasileira para o Desenvolvimento do Ensino de Ciências, 228p.

BOURRELly, P. 1968. Les alges d'eau douce; initiation à la systematique. Les alges jaunes et brunes, Chrysophycées, Theophycées, Xanthophycées et Diatomées. Paris, Ed. N. Bousée \& Cie., vol 2, 438p.

1970. Les alges d'eau douce; initiation à la systematique. Les alges blenes et rouges, les Euglénèens, Peridiniens et Cryptomonadines. Paris, Ed. N. Bousée \& Cie., vol 3, 512p.

1972. Les alges d'eau douce; initiation à la systematique. Les alges vertes 2 ed. Paris, Ed N. Bousée \& Cie. vol. 1, 572p.

BRITTON, R.H. \& M.E. MOSER. 1982. Size specific predation by herons and effect on the sex-ratio of natural populations of the mosquito fish Gambusia affinis Baird and Girard. Oecologia 53: 146-151.

CONNOR, E.F. \& D.S. SimBERLOFF. 1979. The assembly of species communities: change or competition? Ecology 60: 1132-1140.

CosTA, W.J.E.M. 1987. Feeding habits of a fish community in a tropical coastal stream, Rio Mato Grosso, Brazil. Stud. Neotrop. Fauna and Env. 22 (3): 145-153.

DiAMOND, J.M. 1978. Niche shifts and the rediscovery of interspecific competitions. Amer. Sci. 66: 322-331.

EGGERS, D.M. 1977. Planktivorous preference by prey size. Ecology 63 (2): 381-390.

Grossman, G.D.; P.B. MOYLE \& J.O. WHITAKER JR. 1982. Stochascity in structural and functional characteristics of an Indiana stream fish assemblage: A test of community theory. Amer. Nat. 120 (4): 423-454.

HENN, A.W. 1916. On various south american poeciliid fishes. Ann. Carnegie Mus. 10: 93-142.

HoRN, M.H. 1972. The amount of space available for marine and freshwater fishes. Carnegie Mus. 70: 1295-1297.

HunTCHINSON, G.E. 1958. Concluding remarks. Cold Spring Harbor Symp. Quant. Biol. 22: 415-427.

- 1959. Homage to Santa Rosalia, or why are there so many kinds of animals? Amer. Nat. 93: 145-159.

HYSLOP, E.P. 1980. Stomach contents analysis: a review of methods and their application. Jour. Fish. Biol. 17: 411-429.

KAWAKAMI, E. \& G. VAZZOLER. 1980. Método gráfico e estimativa do Índice Alimentar aplicado no estudo de alimentação de peixes. Bolm. Inst. Oceanogr., São Paulo, 29 (2): 205-207.

KEAST, A. 1978. Trophic and spatial interrelationships in the fish species of an Ontario temperate lake. Env. Biol. Fish. 3: 7-31.

KRAMER, D.L. 1978. Reproductive seasonality in the fishes of a tropical stream. Ecology 59 (5): 976-985.

KRUMHOLZ, L.A. 1948. Reprodution in the western mosquito-fish Gambusia affinis (Baird \& Giard), and its use in mosquito control. Ecol. Monog. 18: 1-43.

MACAN, T.T. 1959. Guia de animales invertebrados de agua dulce. Eunsa, Pamplona, 118p. 
MACARTHUR, R.H. 1972. Geographical Ecology. Harper and Row, New York, $269 p$.

MENEZES, M.S. \& E.P. CARAMASCHI. 1994. Características reprodutivas de Hypostomus grupo H. punctatus (Osteichthyes, Siluriformes) no rio Ubatiba, Maricá, RJ. Rev. Brasil. Biol. 54 (3): 503-513.

NARAHARA, M.Y.; H.M. GoDINHO \& E. ROMAGOSA. 1985. Estrutura da população de Rhamdia hilarii (Valenciennes, 1840) (osteichthyes, Siluriformes, Pimelodidade). Bol. Inst. Pesca, São Paulo, 12 (3): 123-137.

NeEdHAm, J.G. \& P.R. NeEdHAM. 1978. Guía para el estudio de los seres vivos de las aguas dulces. Reverté, Barcelona, 131p.

PAYNE, A.L. 1986. The ecology of tropical lakes and rivers. New York, John Wiley \& Sons, 301p.

PiANKA, E.R., 1982. Ecología Evolutiva. New York, Omega S.A., 365p.

PRICE, P.W. 1984. Alternatives paradigms in community ecology, p. 354-377. In: P.W. PRICE \& W.S. SLOBODCHIROF (Eds). A new ecology: Novel approach to interactive systems. New York, John Wiley \& Sons, 515p.

RoMESBURG, H.C. 1990. Cluster analysis for researchers. Malabar, Robert E. Krieger Publishing Company, 334p.

Ross, S.T. 1986. Resource partitioning in fish assemblages: a review of field studies. Copeia (2): 352-388.

SABINO, J. \& R.M.C. CASTRO. 1990. Alimentação, período de atividade e distribuição espacial dos peixes de um riacho da floresta Atlântica (Sudeste do Brasil). Rev. Brasil. Biol. 50 (1): 23-36.

SCHÖENER, T.W. 1974. Resource partitioning in ecological communities. Science 185: $27-38$.

SIEGEL, S. 1975. Estatística não-paramétrica (para ciências do comportamento). São Paulo, McGraw-Hill, 350p.

SMITH, E.P. \& T.M. ZARET. 1982. Bias in estimating niche overlap. Ecology 63 (5): $1248-1253$.

SOARES-PorTO, L.M.S. 1994. Dieta e ciclo diuturno de atividade alimentar de Pimelodella lateristriga (Müller \& Troschel, 1849) (Siluroidei, Pimelodidae) no rio Ubatiba, Maricá, Rio de Janeiro. Rev. Brasil. Biol. 54 (3): 451-458.

StRONG, D.R. 1980. Null hypothesis in ecology. Synthese 43: 271-285.

TEIXEIRA, R.L. 1989. Aspectos da ecologia de alguns peixes do arroio Bom Jardim, Triunfo - Rs. Rev. Brasil. Biol. 49 (1): 183-192.

ThIBAUlT, R.E. \& R.J. SchulTz. 1978. Reprodutive adaptations among viviparous fishes (Cyprinodontiformes: Poeciliidae). Evolution 32 (2): 320-333.

WERNER, E.E.; T.J. HALL \& M.D. WERNER. 1978. Littoral zone fish communities of two Florida lakes and a comparison with Michigan Lake. Env. Biol. Fish. 3: 163-172.

WIENS, J.A. 1977. On competition and variable enviroments. Amer. Sci. 65: 590-597.

YANT, P.R.; J.R. KARR \& P.L. ANGermeIER. 1984. Stochasticity in stream fish communities: an alternative interpretation. Amer. Nat. 124 (4): 573-582. 